\section{PTH-063 VACCINATION AGAINST OPPORTUNISTIC INFECTIONS IN PATIENTS WITH INFLAMMATORY BOWEL DISEASE ON IMMUNOMODULATOR THERAPY}

doi:10.1136/gut.2011.239301.464

C T Tee, ${ }_{1,}^{1}$ S T Peake, ${ }^{2} \mathrm{~J}$ Landy, ${ }^{2}$ N Arebi ${ }^{2}$ 'APRG Imperial College London, London, UK; ${ }^{2}$ Gastroenterology, St Mark's Hospital, London, UK

Introduction Immunomodulators (IM) and biological agents are now used more often and earlier in Inflammatory Bowel Disease (IBD), leading to an increase in opportunistic infections (OI). The RR of OI is reported as OR of 2.9 with one IM, increasing to $O R$ of 14.5 with $\geq 2$ concomitant IMs. Age (>50 years) is also an independent risk factor for OI. These vaccinations are recommended by the European Crohn's and Colitis Organisation (ECCO) to minimise OI in immunocompromised IBD patients; Varicella Zoster Virus (VZV) (if no history of chickenpox/shingles and serology is negative), Human Papilloma Virus (HPV), annual Influenza (inactivated vaccine), Pneumococcal and Hepatitis B (if HBV seronegative). Following the Influenza A outbreak (H1N1) in 2009, Swine Flu vaccination was also recommended. The authors aimed to investigate the vaccination status of IBD patients on one or more IM.

Methods The authors identified a cohort of 200 patients on IM or biological therapy from outpatients attendance between September 2009 and June 2010. Questionnaires were posted to the General Practitioners (GP) to obtain vaccination status for Flu (within 1 year), Pneumococcal (within last 5 years), VZV, HPV and Swine Flu. T test was used to examine for differences in higher risk groups.

Results The response rate was 51.5\% (103/200). Median age was 42 years (range 16-74); 54 were female (52.4\%). 82 were tertiary referrals (79.6\%). 75.7\% had Crohn's disease ( $n=78)$. IMs included Azathioprine (35.9\%, $\mathrm{n}=37$ ), 6-Mercaptopurine $(9.7 \%, \mathrm{n}=10)$, corticosteroids $(5.8 \%, \mathrm{n}=6)$, Methotrexate $(3.8 \%$, $\mathrm{n}=4)$ and Tacrolimus (1.9\%, $\mathrm{n}=2)$. Biological agents included Infliximab (36.9\%, $\mathrm{n}=38)$ and Adalimumab (25.2\%, $\mathrm{n}=26)$.

21 patients were $\geq 50$ years old; $47.6 \%(10 / 21)$ had received the Influenza and/or Swine Flu vaccination compared to $40.2 \%(33 / 82)$ in the younger age group $(p=0.6)$. Of the
10 patients receiving dual IM and biological therapy, 9 (90\%) received both annual Influenza and Swine Flu vaccinations $(\mathrm{p}<0.001)$.

Conclusion Majority of patients at risk of OI in this cohort are on biological therapy. Very few received the recommended vaccinations. Patients on dual immunosuppression had significantly higher vaccine uptake. These findings may be due to lack of awareness of the risks associated with immunosuppression. Information leaflets on the ECCO-recommended vaccines directed at GPs and patients may improve compliance.

Competing interests None.

Keywords inflammatory bowel disease, opportunistic infections, vaccination.

Table 1 PTH-063 Administered vaccinations

\begin{tabular}{|c|c|c|c|c|c|}
\hline \multirow[b]{2}{*}{ Vaccine } & \multirow[b]{2}{*}{ Administered (n (\%) } & \multicolumn{2}{|c|}{ Not administered (n (\%)) } & \multirow[b]{2}{*}{ Unknown status (n (\%)) } & \multirow[b]{2}{*}{ Not eligible (n (\%)) } \\
\hline & & Not invited & Patient refused & & \\
\hline Flu & $38(36.9)$ & $56(54.4)$ & $9(8.7)$ & $0(0)$ & $0(0)$ \\
\hline Pneumococcal & $7(6.8)$ & $91(88.3)$ & $4(3.4)$ & $1(0.9)$ & $0(0)$ \\
\hline HPV & $0(0)$ & $14(13.6)$ & $0(0)$ & $1(0.9)$ & $88(85.4)$ \\
\hline VZV & $0(0)$ & $72(70.0)$ & $0(0)$ & $7(6.7)$ & $24(23.3)^{*}$ \\
\hline
\end{tabular}

*Patient previously contracted illness, not eligible for vaccination. 